\title{
Internalized Weight Bias and Disordered Eating: The Mediating Role of Body Image Avoidance and Drive for Thinness
}

\author{
Rachel D. Marshall*, Janet D. Latner and Akihiko Masuda \\ Department of Psychology, University of Hawai'i at Mānoa, Honolulu, HI, United States
}

Internalized weight bias has been linked with undesirable physical and psychological health outcomes, including disordered eating. Interventions have targeted internalized weight bias and associated outcomes, but little is known about underlying mechanisms of change. Existing treatment literature suggests that drive for thinness and body image avoidance may sustain the link between internalized weight bias and disordered eating. The present study aimed to determine if drive for thinness and body image avoidance mediated the relationship between internalized weight bias and disordered eating in an

OPEN ACCESS

Edited by: Stuart William Flint, University of Leeds, United Kingdom

Reviewed by: Giovanni Maria Ruggiero, Studi Cognitivi S.p.A, Italy

Giulia Corno,

Université du Québec en Outaouais,

Canada

*Correspondence:

Rachel D. Marshall

rachel.marshall@hawaii.edu

Specialty section:

This article was submitted to Health Psychology,

a section of the journal

Frontiers in Psychology

Received: 02 October 2019

Accepted: 18 December 2019

Published: 22 January 2020

Citation:

Marshall RD, Latner JD and Masuda A (2020) Internalized Weight Bias and Disordered Eating: The Mediating Role of Body Image Avoidance and Drive for Thinness.

Front. Psychol. 10:2999.

doi: 10.3389/fpsyg.2019.02999 ethnically diverse sample. Participants included 225 female college students aged 1849 years (mean age $=20.4$ years, $S D=4.4$ ), with a mean BMl of $23.3 \mathrm{~kg} / \mathrm{m}^{2}$ who completed a computer-based survey for partial course credit. As expected, internalized weight bias was positively associated with disordered eating, and results supported the hypothesis of the mediating role of drive for thinness and body image avoidance. These results are important given the shortage of intervention efforts targeting internalized weight bias. Future intervention efforts aimed at reducing internalized weight bias and associated outcomes may benefit from simultaneously targeting drive for thinness and body image avoidance.

Keywords: internalized weight bias, disordered eating, eating disorders, body image, drive for thinness, internalized weight stigma, body image avoidance

\section{INTRODUCTION}

Internalized weight bias (IWB), the belief that negative stereotypes about weight apply to the self, is a significant concern in women, as it has been linked with a range of physical and psychological issues, including disordered eating (Durso et al., 2012, 2016). For example, IWB is associated with binge eating among treatment-seeking adults (Carels et al., 2010) and with dietary restraint among college women (Sienko et al., 2016). Over the course of treatment, IWB may moderate the efficacy of existing health interventions for women who are overweight, as participants with high IWB do not experience improvements in disordered eating (Mensinger et al., 2016).

Contemporary cognitive behavioral therapy (CBT) has attempted to offset the negative impact of IWB (Carels et al., 2014; Pearl et al., 2018). However, evidence regarding the mechanisms of change to offset the link between IWB and disordered eating remains sparse. Testing factors that are theorized to sustain the relationship between IWB and disordered eating is essential for understanding intervention efforts and improving their efficacy. Existing literature on CBT for 
eating disorders suggests that body image avoidance and drive for thinness may be underlying mechanisms of the iatrogenic link between IWB and disordered eating. The present crosssectional study examined whether these two variables mediate the association between IWB and disordered eating.

\section{Drive for Thinness}

According to CBT models, drive for thinness is a major risk factor for eating disorders. In treatment, drive for thinness is often a major target of intervention primarily because it is theorized to be an underlying variable that accounts for a range of eating disorder symptoms (Fairburn et al., 2003). Drive for thinness is associated with disordered eating; for example, individuals with eating pathology (e.g., anorexia nervosa) exhibit higher levels of drive for thinness than healthy controls (Garner et al., 1983). In addition, prior research has demonstrated a significant, positive association between IWB and drive for thinness ( $r=0.47$ and $r=0.56)$ among community samples of adults across the entire BMI spectrum (Durso and Latner, 2008; Pearl and Puhl, 2014). Given these associations, first, between drive for thinness and disordered eating, and second, between IWB and drive for thinness, it can be speculated that drive for thinness might serve as a mediator of the connection between IWB and disordered eating.

\section{Body Image Avoidance}

Body image avoidance is another candidate that might explain the link between IWB and disordered eating. Body image avoidance is often defined as refraining from entering situations that elicit worry about physical appearance (Rosen et al., 1991). Examples of body image avoidance include avoiding reflective surfaces (e.g., mirrors), being weighed, wearing formfitting clothes, and being photographed. Body image avoidance is an important behavioral manifestation of body image disturbance, which includes cognitive, affective, and behavioral expressions of body dissatisfaction (Stormer and Thompson, 1996; Pellizzer et al., 2018). Multiple studies have observed a positive association between body image avoidance, disordered eating, and negative attitudes about body weight and shape (Durso and Latner, 2008; Walker et al., 2018; Purton et al., 2019). Additionally, higher IWB is associated broadly with body image disturbances (Durso and Latner, 2008; Carels et al., 2010; Durso et al., 2016). Considering the existing associations between body image avoidance with disordered eating and body image disturbance with IWB, there is reason to predict that a behavioral expression of body image disturbance (i.e., body image avoidance) mediates the relationship between IWB and disordered eating.

\section{Drive for Thinness and Body Image Avoidance as Two Underlying Mechanisms}

Cognitive behavioral therapy literature emphasizes drive for thinness and body image avoidance as variables associated with both IWB and disordered eating. IWB significantly predicted drive for thinness among an overweight community sample (Durso and Latner, 2008). Additionally, a transdiagnostic model of eating disorders emphasized drive for thinness as a risk factor and maintaining factor for eating disturbances (Fairburn et al., 2003). Body image avoidance is an important behavioral manifestation of body image disturbance, which prior research indicates is positively associated with IWB (Durso and Latner, 2008; Carels et al., 2010). Amin et al. (2014) observed greater body image avoidance among a clinical sample with eating disorders than a non-clinical control group. Additionally, reduction in body image avoidance is a significant predictor of improvement in disordered eating (Pellizzer et al., 2019). Considering aforementioned findings, both drive for thinness and body image avoidance should be simultaneously explored as potential mediators of the relationship between IWB and disordered eating. Drive for thinness and body image avoidance are associated cognitive and behavioral factors, both of which have been independently linked with IWB and disordered eating behaviors. For example, a woman who reports high levels of IWB is likely to experience a strong drive for thinness, along with behavioral efforts to avoid or down-regulate her negative body image. As potential underlying mechanisms in the context of IWB, both the urge to become thinner and efforts to down-regulate negative body image may trigger and perpetuate symptoms of disordered eating.

\section{Present Study}

Existing literature has demonstrated associations between IWB, disordered eating, drive for thinness, and body image avoidance. CBT models suggest that drive for thinness and body image avoidance are underlying mechanisms of eating disorder pathology that are activated in the context of IWB, which in turn results in greater disordered eating. Although researchers have examined associations among variables of present interest partially, the proposed multiple mediation model has not been fully investigated. The present cross-sectional study was designed to directly investigate the proposed multiple mediation model.

\section{METHOD}

\section{Participants}

Female students were recruited from a public university in Hawaii through an online research recruitment pool managed by the Department of Psychology. To be included in this study, participants had to identify as female, be 18 or older, and speak English.

\section{Procedure and Measures}

The present research was approved by and was carried out in accordance with the recommendations of the University of Hawaii Institutional Review Board. All subjects gave written informed consent prior to participation in this study. Participants who enrolled in the study were asked to complete an anonymous online survey, including survey measures and demographic information. The following measures were used to assess internalized weight bias, disordered eating, drive for thinness, and body image avoidance. 


\section{Internalized Weight Bias}

The Weight Bias Internalization Scale - Modified (WBISM; Pearl and Puhl, 2014) is an 11-item self-report measure of IWB. This measure is a revised version of the original Weight Bias Internalization Scale (WBIS) by Durso and Latner (2008). Responses were rated on a 7-point Likert scale ranging from "Strongly Disagree" to "Strongly Agree". The IWBS-M demonstrated high internal consistency $(\alpha=0.90)$ and was positively correlated with BMI in a validation study (Pearl and Puhl, 2014). Similarly, the WBIS-M demonstrated high internal consistency $(\alpha=0.95)$ and was significantly correlated with BMI $(r=0.47, p<0.001)$ in the present study.

\section{Disordered Eating}

The Eating Attitudes Test (EAT-26; Garner et al., 1982) is a 26item self-report measure used to detect symptoms and concerns related to disordered eating. Responses were rated on a 6-point Likert scale ranging from "Always" to "Never". The EAT-26 demonstrated high reliability in a study of its psychometric properties among females with $(\alpha=0.90)$ and without $(\alpha=0.83)$ anorexia nervosa (AN; Garner et al., 1982). Additionally, the EAT-26 was highly correlated with the original 40-item scale, EAT-40 (Garner et al., 1982). In the present study, the EAT-26 had high internal consistency $(\alpha=0.88)$.

\section{Drive for Thinness}

The Eating Disorders Inventory - Drive for Thinness Subscale (EDI- Drive for Thinness; Garner et al., 1983) is a 7-item selfreport scale that measures fear of weight gain and desire to lose weight. Responses were rated on a 6-point Likert scale ranging from "Always" to "Never". The scale demonstrated high internal consistency among those who met criteria for AN $(\alpha=0.85)$ and a female control sample ( $\alpha=0.85$; Garner et al., 1983). Internal consistency of the EDI-Drive for thinness Subscale was high in the present sample $(\alpha=0.92)$.

\section{Body Image Avoidance}

The Body Image Avoidance Questionnaire (BIAQ; Rosen et al., 1991) is 19-item self-report questionnaire created to measure avoidance of situations that elicit concern about physical appearance. Responses were rated on a 6-point Likert scale ranging from "Never" to "Always". In a validation study of the BIAQ among female university students, the scale demonstrated good internal consistency ( $\alpha=0.89$; Rosen et al., 1991). The questionnaire also had good internal consistency $(\alpha=0.84)$ in the present sample.

\section{Data Analysis}

Descriptive statistics were run on demographic information to summarize the age, ethnicity, relationship status, and weight history of participants in our sample. We calculated the mean and standard deviation for all scales, and then examined the nature of the associations among the variables using bivariate correlations.

In order to examine the indirect effect of internalized weight bias on disordered eating through drive for thinness and body image avoidance, separately, a mediation analysis was conducted using a bootstrapping procedure based on the recommendation of A. F. Hayes (2013) and MacKinnon et al. (2007). The parallel mediation analysis was computed using the PROCESS macro for SPSS, version 3.4 (A. F. Hayes, 2013). The bootstrapping procedure constructed bias-corrected confidence intervals based on 5,000 random samples with replacement from the full sample (see: Preacher and Hayes, 2004, 2008). Direct effects, indirect effects, standard errors, and confidence intervals were estimated based on the distribution obtained using the bootstrapping procedure. A significant indirect effect indicates that the effect of IWB on the outcome variable disordered eating is accounted for by a mediating variable (e.g., drive for thinness), while controlling for the other mediator (e.g., body image avoidance).

\section{RESULTS}

Participants $(N=294)$ were undergraduate women enrolled in introductory psychology classes who completed the present web-based survey. Of those, 36 participants were excluded from the study because they did not identify as female. An additional 20 participants were excluded from the study because they were under 18-years-old. In order to determine how to handle missing data, Little's Missing Completely at Random test (Little, 1988) was run $X^{2}(441, N=225)=447.28, p=0.408$. This non-significant finding indicates that data were missing completely at random and the pattern of missing values does not depend on data values. Thus, listwise deletion of missing data from 13 participants who were missing partial data was deemed appropriate and unlikely to bias study results. The final sample consisted of 225 participants ranging in age from 18 to 49 years old $(M=20.39, S D=4.41)$ and ranging in BMI from 15.58 to $40.72(M=23.29, S D=4.92)$. The sample identified as follows: $45.8 \%$ Asian $(n=103), 31.6 \%$ Caucasian $(n=71), 8 \%$ Native Hawaiian/Pacific Islander $(n=18), 2.7 \%$ Multiracial $(n=6), 2.2 \%$ Hispanic/Latina $(n=5), 1.8 \%$ African American $(n=4)$, and $6.2 \%$ other ethnicity $(n=14)$. The sample was reflective of the ethnic diversity of the city in which the research was conducted.

\section{Associations Among Study Variables}

Pearson correlations were calculated among the study variables (see Table 1). IWB was positively correlated with disordered eating, drive for thinness, body image avoidance, and BMI. Additionally, disordered eating was positively correlated with drive for thinness, body image avoidance, and BMI. Drive for thinness was positively associated with body image avoidance and BMI. Last, body image avoidance was positively correlated with BMI.

\section{Mediation Analysis}

A parallel mediation analysis was conducted using a bootstrapping resampling procedure to examine the indirect effect of IWB on disordered eating through drive for thinness and body image avoidance (see Figure 1). The size of the indirect effect of IWB on disordered eating through drive for thinness was $0.22(S E=0.04)$, and it was significant with a 95\% confidence interval which did not include zero, 
TABLE 1 | Pearson correlations between study variables.

\begin{tabular}{|c|c|c|c|c|c|}
\hline Variable & 1 & 2 & 3 & 4 & 5 \\
\hline 1. Internalized weight bias (WBIS-M) & - & & & & \\
\hline 2. Disordered eating (EAT-26) & $0.54^{\star \star}$ & - & & & \\
\hline 3. Drive for thinness (EDI subscale) & $0.67^{\star \star}$ & $0.69^{\star \star}$ & - & & \\
\hline 4. Body image avoidance (BIAQ) & $0.63^{\star \star}$ & $0.58^{\star \star}$ & $0.58^{\star *}$ & - & \\
\hline 5. BMl & $0.47^{\star \star}$ & $0.22^{*}$ & $0.32^{\star \star}$ & $0.23^{\star \star}$ & - \\
\hline Mean & 37.65 & 10.09 & 5.15 & 27.54 & 23.29 \\
\hline SD & 15.77 & 10.10 & 6.02 & 11.10 & 4.92 \\
\hline
\end{tabular}

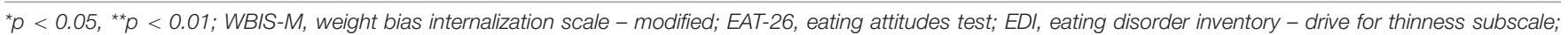
$B I A Q$, body image avoidance questionnaire; BMI, body mass index; SD, standard deviation.

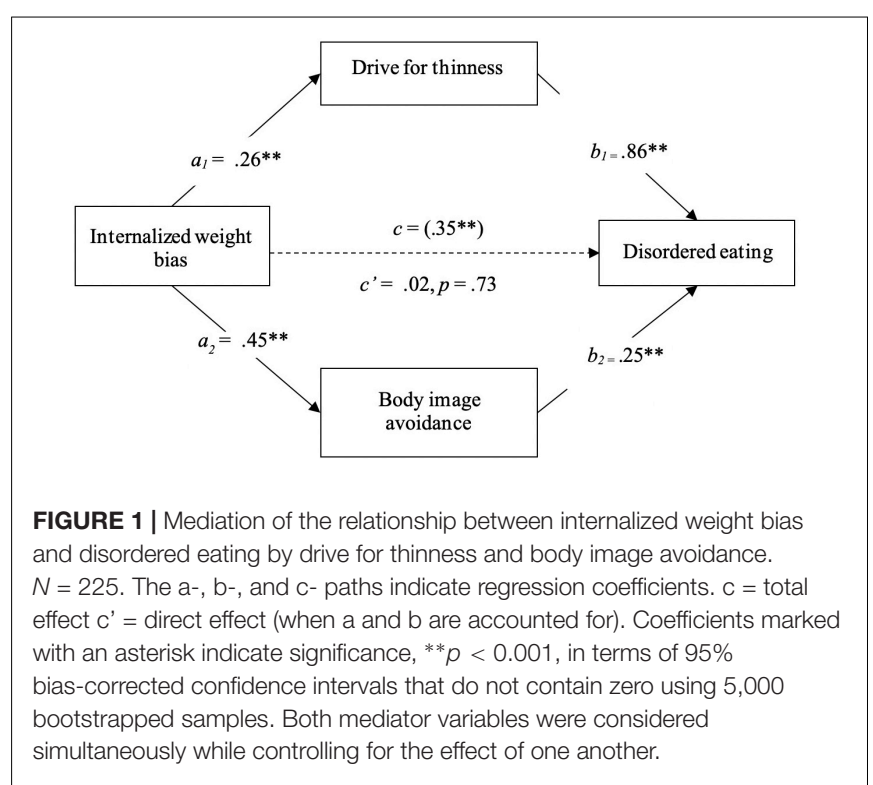

95\% CI $[0.15,0.30]$. This result indicates that the effect of IWB on disordered eating is mediated by drive for thinness. The size of the indirect effect of IWB on disordered eating through body image avoidance was $0.11(S E=0.03)$, and it was significant with a $95 \%$ confidence interval which did not include zero, 95\% CI $[0.05,0.17]$. This result indicates that the effect of IWB on disordered eating is also mediated by body image avoidance. The mediators, body image avoidance and drive for thinness, demonstrated significant a-paths and b-paths. The total effect of IWB on disordered eating (c-path) was reduced to non-significance (c'-path) when controlling for the effects of the mediators. Results indicate full parallel mediation of the relationship between IWB and disordered eating by drive for thinness and body image avoidance. In other words, the association between IWB and disordered eating is fully accounted for by drive for thinness and body image avoidance.

\section{DISCUSSION}

Internalized weight bias is cause for concern due to its association with numerous undesirable psychological and physical health issues, such as low self-esteem and disordered eating. Disordered eating is one health outcome of present interest, given the prevalence and persistence of eating disturbances among college women (Eisenberg et al., 2011). Little is known about mechanisms of change in interventions that attempt to reduce IWB and associated disordered eating. Guided by CBT models of eating disorders and eating disorder treatment, the present study was designed to determine whether drive for thinness and body image avoidance mediated the relationship between IWB and disordered eating. IWB, disordered eating, drive for thinness, body image avoidance, and BMI were positively associated with each other. Results support previous findings indicating positive association between IWB and disordered eating (e.g., binge eating) among samples of community and college adults (Puhl et al., 2007; Durso et al., 2016; Mehak et al., 2018). Findings also suggest the potential generalizability of such associations to subgroups of ethnically diverse women who are often not included in studies of body image and eating concerns.

A multiple mediation analysis showed that body image avoidance and drive for thinness together mediated the relationship between IWB and disordered eating, providing support for our hypotheses. These findings demonstrated that the relationship between IWB and disordered eating was not direct. Rather, the association between IWB and disordered eating was fully accounted for by other variables in this sample, namely, drive for thinness and body image avoidance. This finding is novel and important given the shortage of studies designed to reduce IWB and associated negative outcomes. Knowing the factors that maintain the relationship between IWB and disordered eating elucidates ideas for identifying or creating a potent intervention that addresses mediating factors in addition to IWB.

The current research lends itself to suggestions for future research. The present study included an ethnically diverse sample, including Asian, Caucasian, Native Hawaiian/Pacific Islander, Hispanic/Latina, and multiracial participants, but multiple groups were too small to permit conducting analyses by individual ethnic group. Examining the present mediation model across ethnic groups would allow researchers who work with diverse populations to confidently conceptualize relationships between variables of interest among those individuals. Future research would do well to recruit larger 
numbers of ethnically diverse individuals in order to facilitate greater generalizability of findings.

Findings from the current study have clinical implications for future prevention and intervention efforts targeting IWB and its association with eating disorder pathology. One might consider how the four variables of interest are experienced by a patient. A woman who attributes negative stereotypes (e.g., lazy, undisciplined) to herself due to her size or shape will likely avoid stimuli that trigger her body dissatisfaction while simultaneously making efforts to pursue a thinner body. In order to lose weight, the woman may avoid eating calorically dense foods with low nutritional value prior to experiencing unwanted binge eating episodes. This entire experience will reinforce the woman's belief that she is undisciplined. Since drive for thinness and body image avoidance fully explain the association between IWB and disordered eating, interventions designed to prevent and/or reduce disordered eating among patients may be strengthened by explicitly targeting the mediators in addition to IWB. Such a multifaceted treatment would be closely aligned with cognitive-behavioral and acceptance-based intervention models, such as CBT, CBT-Enhanced (CBT-E; Byrne et al., 2011) and acceptance and commitment therapy (ACT; S. C. Hayes et al., 2006). CBT and ACT target IWB and disordered eating by undermining the negative impact of body image avoidance and drive for thinness. In light of current findings, use of interventions that challenge negative weight-based stereotypes (Carels et al., 2014), encourage body acceptance (Pearl et al., 2018), and facilitate cognitive defusion (i.e., identifying thoughts as products of the mind and not necessarily the truth; Lillis et al., 2009; Palmeira et al., 2017) may be beneficial for reducing IWB and associated negative outcomes.

Interventions for IWB often focus on individuals struggling with overweight or obesity (Berman et al., 2016; Levin et al., 2018; Pearl et al., 2018). Results from the present study show that while greater IWB is associated higher BMI scores, IWB also exists among females who are not overweight. This result is in line with other recent findings (Essayli et al., 2017), and indicates that females of varying sizes struggle with self-judgment based on their weight and shape. Women across the weight spectrum may be suffering from the deleterious psychological and physical impacts of IWB, including disordered eating. Future clinical interventions would do well to address IWB among individuals across the body weight spectrum.

Our findings should be interpreted in light of several notable limitations. First, the research explored a non-clinical sample. It is unknown if variables in the present study function similarly among those with clinical levels of eating disturbances. Additionally, an all-female sample was used because the variables of interest are thought to function differently between women and men (Smith et al., 2017; Puhl et al., 2018; Purton et al., 2019). The generalizability of the present findings to men is unknown. Future research that examines the relationship between IWB and disordered eating in men is encouraged. Additionally, the present study included a wider range of ages than is found in many college samples; however, the generalizability of the present findings to females under 18 or over 50 remains unclear. Another limitation includes the use of self-report measures. Participants may have been impacted by the social desirability bias, underreporting symptoms in order to present themselves favorably. The present study was correlational in nature, and for this reason, causal inferences cannot be made. Longitudinal and experimental research is needed to establish causation and determine the long-term effects of change in a particular variable (e.g., IWB).

The present study contributes to the study of IWB, body image, and disordered eating in meaningful ways. It confirms previously established associations among key variables in an ethnically diverse sample. Additionally, the current research contributes to the understanding of the relationship between IWB and disordered eating by indicating that drive for thinness and body image avoidance fully account for their association. CBT and ACT have independently demonstrated efficacy in reducing body image avoidance and drive for thinness, and both treatment modalities have also demonstrated preliminary success in reducing IWB. Future intervention efforts might do well to utilize CBT or ACT to target IWB, body image avoidance, and drive for thinness to prevent and/or reduce disordered eating.

\section{DATA AVAILABILITY STATEMENT}

The raw data supporting the conclusions of this article will be made available by the authors, without undue reservation, to any qualified researcher.

\section{ETHICS STATEMENT}

The studies involving human participants were reviewed and approved by the University of Hawai'i at Mānoa Institutional Review Board. The participants provided their written informed consent to participate in this study.

\section{AUTHOR CONTRIBUTIONS}

RM co-designed and conducted the study, analyzed the data, and wrote the manuscript. JL co-designed the study and edited the manuscript. AM collaborated on writing and editing the manuscript.

\section{FUNDING}

The authors received funding from the University of Hawai' $i$ at Mānoa, Department of Psychology for open access publication fees. The funders had no role in study design, data collection and analysis, decision to publish, or preparation of the manuscript. 


\section{REFERENCES}

Amin, R., Strauss, C., and Waller, G. (2014). Body-related behaviours and cognitions in the eating disorders. Behav. Cogn. Psychother. 42, 65-73. doi: 10.1017/S1352465812000914

Berman, M. I., Morton, S. N., and Hegel, M. T. (2016). Uncontrolled pilot study of an acceptance and commitment therapy and health at every size intervention for obese, depressed women: accept yourself! Psychotherapy 53, 462-467. doi: $10.1037 /$ pst0000083

Byrne, S. M., Fursland, A., Allen, K. L., and Watson, H. (2011). The effectiveness of enhanced cognitive behavioural therapy for eating disorders: an open trial. Behav. Res. Ther. 49, 219-226. doi: 10.1016/j.brat.2011.01.006

Carels, R. A., Burmeister, J. M., Koball, A. M., Oehlhof, M. W., Hinman, N., Leroy, M., et al. (2014). A randomized trial comparing two approaches to weight loss: differences in weight loss maintenance. J. Health Psychol. 19, 296-311. doi: $10.1177 / 1359105312470156$

Carels, R. A., Wott, C. B., Young, K. M., Gumble, A., Koball, A., and Oehlhof, M. W. (2010). Implicit, explicit, and internalized weight bias and psychosocial maladjustment among treatment-seeking adults. Eat. Behav. 11, 180-185. doi: 10.1016/j.eatbeh.2010.03.002

Durso, L. E., and Latner, J. D. (2008). Understanding self-directed stigma: development of the weight bias internalization scale. Obesity 16(Suppl. 2), S80-S86. doi: 10.1038/oby.2008.448

Durso, L. E., Latner, J. D., and Ciao, A. C. (2016). Weight bias internalization in treatment-seeking overweight adults: psychometric validation and associations with self-esteem, body image, and mood symptoms. Eat. Behav. 21, 104-108. doi: 10.1016/j.eatbeh.2016.01.011

Durso, L. E., Latner, J. D., White, M. A., Masheb, R. M., Blomquist, K. K., Morgan, P. T., et al. (2012). Internalized weight bias in obese patients with binge eating disorder: associations with eating disturbances and psychological functioning. Int. J. Eat. Disord. 45, 423-427. doi: 10.1002/eat.20933

Eisenberg, D., Nicklett, E. J., Roeder, K., and Kirz, N. E. (2011). Eating disorder symptoms among college students: prevalence, persistence, correlates, and treatment-seeking. J. Am. College Health 59, 700-707. doi: 10.1080/07448481. 2010.546461

Essayli, J. H., Murakami, J. M., Wilson, R. E., and Latner, J. D. (2017). The impact of weight labels on body image, internalized weight stigma, affect, perceived health, and intended weight loss behaviors in normal-weight and overweight college women. Am. J. Health Promot. 31, 484-490. doi: 10.1177/ 0890117116661982

Fairburn, C. G., Cooper, Z., and Shafran, R. (2003). Cognitive behaviour therapy for eating disorders: a "transdiagnostic" theory and treatment. Behav. Res. Ther. 41, 509-528. doi: 10.1016/S0005-7967(02)00088-8

Garner, D. M., Olmsted, M. P., Bohr, Y., and Garfinkel, P. E. (1982). The Eating Attitudes Test: psychometric features and clinical correlates. Psychol. Med. 12, 871-878. doi: 10.1017/S0033291700049163

Garner, D. M., Olmsted, M. P., and Polivy, J. (1983). Development and validation of a multidimensional eating disorder inventory for anorexia nervosa and bulimia. Int. J. Eat. Disord. 2, 15-34. doi: 10.1002/1098-108x(198321)2:2<15:: aid-eat2260020203>3.0.co;2-6

Hayes A. F. (2013). Introduction to Mediation, Moderation, and Conditional Process Analysis: A Regression-Based Approach. New York: Guilford Press.

Hayes, S. C., Luoma, J. B., Bond, F. W., Masuda, A., and Lillis, J. (2006). Acceptance and commitment therapy: model, processes and outcomes. Behav. Res. Ther. 44, 1-25. doi: 10.1016/j.brat.2005.06.006

Levin, M. E., Potts, S., Haeger, J., and Lillis, J. (2018). Delivering acceptance and commitment therapy for weight self-stigma through guided self-help: results from an open pilot trial. Cogn. Behav. Pract. 25, 87-104. doi: 10.1016/j.cbpra. 2017.02.002

Lillis, J., Hayes, S. C., Bunting, K., and Masuda, A. (2009). Teaching acceptance and mindfulness to improve the lives of the obese: a preliminary test of a theoretical model. Ann. Behav. Med. 37, 58-69. doi: 10.1007/s12160-009-9083-x

Little, R. J. A. (1988). A Test of Missing Completely at Random for Multivariate Data with Missing Values. J. Am. Stat. Assoc. 83, 1198-1202. doi: 10.1080/ 01621459.1988.10478722

MacKinnon, D. P., Fairchild, A. J., and Fritz, M. S. (2007). Mediation analysis. Ann. Rev. Psychol. 58:593.

Mehak, A., Friedman, A., and Cassin, S. E. (2018). Self-objectification, weight bias internalization, and binge eating in young women: testing a mediational model. Body Image 24, 111-115. doi: 10.1016/j.bodyim.2018. 01.002

Mensinger, J. L., Calogero, R. M., and Tylka, T. L. (2016). Internalized weight stigma moderates eating behavior outcomes in women with high BMI participating in a healthy living program. Appetite 102, 32-43. doi: 10.1016/j. appet.2016.01.033

Palmeira, L., Pinto-Gouveia, J., and Cunha, M. (2017). Exploring the efficacy of an acceptance, mindfulness \& compassionate-based group intervention for women struggling with their weight (Kg-Free): a randomized controlled trial. Appetite 112, 107-116. doi: 10.1016/j.appet.2017.01.027

Pearl, R. L., Hopkins, C., Berkowitz, R., and Wadden, T. (2018). Group cognitivebehavioral treatment for internalized weight stigma: a pilot study. Eat. Weight Disord. 23, 357-362. doi: 10.1007/s40519-016-0336-y

Pearl, R. L., and Puhl, R. M. (2014). Measuring internalized weight attitudes across body weight categories: validation of the Modified Weight Bias Internalization Scale. Body Image 11, 89-92. doi: 10.1016/j.bodyim.2013.09.005

Pellizzer, M. L., Tiggemann, M., Waller, G., and Wade, T. D. (2018). Measures of body image: confirmatory factor analysis and association with disordered eating. Psychol. Assess. 30, 143-153. doi: 10.1037/pas0000461

Pellizzer, M. L., Waller, G., and Wade, T. D. (2019). Predictors of outcome in cognitive behavioural therapy for eating disorders: an exploratory study. Behav. Res. Ther. 116, 61-68. doi: 10.1016/j.brat.2019.02.005

Preacher, K. J., and Hayes, A. F. (2004). SPSS and SAS procedures for estimating indirect effects in simple mediation models. Behav. Res. Methods Instrum. Comput. 36, 717-731. doi: 10.3758/BF03206553

Preacher, K. J., and Hayes, A. F. (2008). Asymptotic and resampling strategies for assessing and comparing indirect effects in multiple mediator models. Behav. Res. Methods 40, 879-891. doi: 10.3758/brm.40.3.879

Puhl, R. M., Himmelstein, M. S., and Quinn, D. M. (2018). Internalizing weight stigma: prevalence and sociodemographic considerations in us adults. Obesity 26, 167-175. doi: 10.1002/oby.22029

Puhl, R. M., Moss-Racusin, C. A., and Schwartz, M. B. (2007). Internalization of weight bias: implications for binge eating and emotional well-being. Obesity 15 , 19-23. doi: 10.1038/oby.2007.521

Purton, T., Mond, J., Cicero, D., Wagner, A., Stefano, E., Rand-Giovannetti, D., et al. (2019). Body dissatisfaction, internalized weight bias and quality of life in young men and women. Qual. Life Res. 28, 1825-1833. doi: 10.1007/s11136019-02140-w

Rosen, J. C., Srebnik, D., Saltzberg, E., and Wendt, S. (1991). Development of a Body Image Avoidance Questionnaire. J. Consult. Clin. Psychol. 3, 32-37. doi: 10.1037/1040-3590.3.1.32

Sienko, R. M., Saules, K. K., and Carr, M. M. (2016). Internalized weight bias mediates the relationship between depressive symptoms and disordered eating behavior among women who think they are overweight. Eat. Behav. 22, 141144. doi: 10.1016/j.eatbeh.2016.06.002

Smith, K. E., Mason, T. B., Murray, S. B., Griffiths, S., Leonard, R. C., Wetterneck, C. T., et al. (2017). Male clinical norms and sex differences on the Eating Disorder Inventory (EDI) and Eating Disorder Examination Questionnaire (EDE-Q). Int. J. Eat. Disord. 50, 769. doi: 10.1002/eat.22716

Stormer, S. M., and Thompson, J. K. (1996). Explanations of body image disturbance: a test of maturational status, negative verbal commentary, social comparison, and sociocultural hypotheses. Int. J. Eat. Disord. 19, 193-202. doi: 10.1002/(SICI)1098-108X(199603)19:2<193::AID-EAT10>3.0.CO;2-W

Walker, D. C., White, E. K., and Srinivasan, V. J. (2018). A meta-analysis of the relationships between body checking, body image avoidance, body image dissatisfaction, mood, and disordered eating. Int. J. Eat. Disord. 51, 745-770. doi: $10.1002 /$ eat.22867

Conflict of Interest: The authors declare that the research was conducted in the absence of any commercial or financial relationships that could be construed as a potential conflict of interest.

Copyright (c) 2020 Marshall, Latner and Masuda. This is an open-access article distributed under the terms of the Creative Commons Attribution License (CC BY). The use, distribution or reproduction in other forums is permitted, provided the original author(s) and the copyright owner(s) are credited and that the original publication in this journal is cited, in accordance with accepted academic practice. No use, distribution or reproduction is permitted which does not comply with these terms. 